\title{
Do different attention capture paradigms measure different types of capture?
}

\author{
Nelson A. Roque ${ }^{1} \cdot$ Timothy J. Wright ${ }^{1} \cdot$ Walter R. Boot $^{1}$
}

Published online: 5 May 2016

(C) The Psychonomic Society, Inc. 2016

\begin{abstract}
When something captures our attention, why does it do so? This topic has been hotly debated, with some arguing that attention is captured only by salient stimuli (bottom-up view) and others arguing capture is always due to a match between a stimulus and our goals (top-down view). Many different paradigms have provided evidence for 1 view or the other. If either of these strong views are correct, then capture represents a unitary phenomenon, and there should be a high correlation between capture in these paradigms. But if there are different types of capture (top-down, bottom-up), then some attention capture effects should be correlated and some should not. In 2 studies, we collected data from several paradigms used in support of claims of top-down and bottomup capture in relatively large samples of participants. Contrary to either prediction, measures of capture were not strongly correlated. Results suggest that capture may in fact be strongly determined by idiosyncratic task demands and strategies. Relevant to this lack of relations among tasks, we observed that classic measures of attention capture demonstrated low reliability, especially among measures used to support bottomup capture. Implications for the low reliability of capture measures are discussed. We also observed that the proportion of participants demonstrating a pattern of responses consistent with capture varied widely among classic measures of capture. Overall, results demonstrate that, even for relatively simple
\end{abstract}

Electronic supplementary material The online version of this article (doi:10.3758/s13414-016-1117-4) contains supplementary material, which is available to authorized users.

Nelson A. Roque roque@psy.fsu.edu

1 Department of Psychology, Florida State University, 1107 W. Call St, Tallahassee, FL 32306, USA laboratory measures of attention, there are still important gaps in knowledge regarding what these paradigms measure and how they are related.

Keywords Attentional capture $\cdot$ Attention

The allocation of attention represents a balancing act between the need for goal-directed processing and the need for immediate interruption (attention capture) should the environment change in an important way. Although it is easy to set up situations in the laboratory in which attention is captured, it is harder to answer the question of why attention is captured in these situations. This question has been actively debated for more than 2 decades. Researchers with a "bottom-up" or "stimulus-driven" perspective make the case that it is the visual uniqueness of these attention-capturing stimuli alone that induces capture (e.g., Theeuwes, 1992, 1994; Theeuwes, Kramer, Hahn, \& Irwin, 1998; Yantis \& Jonides, 1984). That is, capture is automatic, unavoidable, and is not dependent upon the observer's current goals and intentions. This view is supported by capture occurring, oftentimes, in situations in which the capturing feature is never associated with the target, observers are told of this fact, and observers are given a valid feature with which to guide their attention to the target. Yet, attention is captured and capture can continue to occur for hundreds of trials or more. Proponents of the stimulus-driven account argue that during the first sweep of attention, attention always first goes to the most salient part of a scene, regardless of the observer's goals.

However, in a series of influential papers, Folk and colleagues questioned this stimulus-driven, bottom-up account of capture (e.g., Folk \& Remington, 1998; Folk, Remington, \& Johnston, 1992). They argued that when attention is captured, it is always due to some match between the observer's 
current goals and the attention-capturing stimulus (e.g., a red distractor may capture attention when searching for a red target, but not an equally salient green distractor). Capture only occurs when a distractor is consistent with an observer's "attention set," or top-down goals. This view is difficult to reconcile from a stimulus-driven perspective; the most salient item in the display should capture attention regardless of the observer's goal.

Researchers with a top-down, or contingent capture, perspective on attention capture have a different view of the results reported by Theeuwes (1992) and derived from similar paradigms. Instead of supporting bottom-up capture, results can also be explained by participants adopting a top-down attention set for uniqueness. This sometimes results in their attention going to the uniquely shaped target (correctly) and sometimes to the salient but irrelevant red distractor (incorrectly). This strategy may be easy to implement and may not result in a large cost, given the quickness with which attention can be reallocated. Supporting this view, adding other shapes to the display so observers must search for the exact shape that defines the target (instead of the uniqueness of the target) eliminates the ability of the red item to capture attention (Bacon \& Egeth, 1994; Leber \& Egeth, 2006b). Furthermore, participants trained to search under a heterogonous shape condition demonstrated no capture when they were subsequently asked to search the same displays Theeuwes (1992) presented participants, suggesting that capture cannot be stimulus driven (Leber \& Egeth, 2006a; see Boot, Brockmole, \& Simons, 2005, for another case in which the same visual stimuli can capture or not capture attention).

While bottom-up capture effects can be reinterpreted as topdown effects, contingent capture effects can also be reinterpreted in a bottom-up framework. In the contingent capture paradigm, a color singleton only captures attention when participants are searching for a color singleton target, and an onset cue will only capture attention when participants are searching for an onset target. Theeuwes (2010) argued that both onset and color singleton cues capture attention regardless of the observer's goal, but attention will only linger at the cue location when the cue matches the observer's attention set (see Blakely, Wright, Dehili, Boot, \& Brockmole, 2012; Boot \& Brockmole, 2010, for similar effects). Color singleton cues will capture attention even when participants are searching for onset targets, but attention will quickly be disengaged because the capturing stimulus is dissimilar from the target. This results in little cost. However, an onset cue would both capture attention and cause attention to dwell for a period of time at that location when participants searched for onsets, resulting in an observable cost. According to Theeuwes (2010), the most salient item in the display always captures attention, and it is only attentional disengagement that is under top-down control.

Despite attempts to reframe effects, some capture paradigms have consistently been used to support top-down, contingent explanations of capture (Folk, Leber, \& Egeth, 2002; Folk, Remington, \& Johnston, 1992) and some have been used to support bottom-up, stimulus-driven explanations of capture (Theeuwes, 1992; Yantis \& Jonides, 1984). These paradigms differ in many respects (e.g., search goal, stimuli, timing, instructions, distractor validity, performance measures). The presented research focused on the interrelationships between capture in these different paradigms to explore the nature of attention capture in these influential paradigms used to support different views. There are three distinct possibilities when it comes to explaining attention capture effects: (1) different capture paradigms measure different types of capture (top down vs. bottom up); (2) all capture paradigms measure bottom-up, stimulus-driven capture; and (3) all capture paradigms measure top-down, contingent capture. In this paper, we address the question of whether capture paradigms measure a unified construct or whether top-down and bottomup paradigms in fact measure different things. Are capture scores that are derived from paradigms purporting to measure stimulus-driven capture more correlated to one another compared to capture scores from paradigms purporting to measure top-down or contingent capture? Or is capture in all paradigms correlated equally, suggesting only one form of capture?

Similar questions have been asked regarding whether measures of spatial capture (e.g., capture in visual search paradigms) relate to temporal capture (capture demonstrated in rapid serial visual presentation paradigms, such as the attention blink paradigm) and have found little relationship between capture in these different paradigms (Kawahara \& Kihara, 2011). This surprising finding, perhaps, supports the idea that the specific demands of a task drive capture and that capture may not be a unitary phenomenon. In our study, we also include both temporal and spatial measures of capture, including two paradigms that both have been thought to measure top-down capture that varied along this dimension. In this way, our results can help tease apart whether a lack of correlation might be because of the differences between spatial and temporal measures of capture or the fact that some paradigms measure top-down capture and others bottom-up capture. We also included multiple search paradigms thought to measure spatial capture. If the distinction between spatial and temporal capture is driving a lack of relationship in this previous study, then capture scores in these paradigms in the current study are predicted to be highly correlated.

The current paper explored these questions in two relatively large samples (Experiment 1, $N=145$; Experiment 2, $N=114$ ). Each of these samples was asked to complete a mixture of paradigms assessing attention capture. In terms of paper structure, first we present the paradigms and demonstrate that each replicates classic attention-capture effects. Afterward, we explore individual differences in capture and how capture scores derived from each paradigm relate to one another. 


\section{Experiment 1}

\section{Method}

\section{Participants}

One hundred forty-six undergraduate students with selfreported normal color vision participated in this study for course credit. This sample had an average age of 20 years $(S D=2.89)$. The present study was powered to detect a correlation of .23 or higher ( $80 \%$ probability), as per a power analysis using G*Power (Faul, Erdfelder, Buchner, \& Lang, 2009). This is a conservative estimate; if these tasks are indeed measuring similar constructs, we expect a correlation to be medium to large.

\section{Materials}

All stimuli for this study were presented on a 19-inch color monitor $(1,024 \times 768$ resolution), with response latency and accuracy collected using a standard keyboard. Observers were seated approximately $60 \mathrm{~cm}$ away from the monitor. Each task used in this study is described in more detail below.

\section{Overview}

In Experiment 1, our approach was to have participants complete variants of two of the most common paradigms used in support of bottom-up capture effects and two of the most common paradigms used in support of top-down, contingent capture effects. Capture scores were then derived in a manner consistent with the literature for each task. For search tasks involving search for a target of a specific color, the color of the search target was held constant (green) to reduce the possibility of carryover effects. All tasks described below included a short practice phase, which was not included in analyses.

\section{Top-down tasks}

\section{Contingent cuing paradigm (based on Folk, Remington, \&}

Johnston, 1992) For this task (created using PsychoPy; Peirce, 2007), participants observed a display featuring five white boxes $\left(4.14^{\circ} \times 5.73^{\circ}\right)$ on a black background. One box was presented centrally, and four boxes were presented 9.17 degrees from the center of the screen (see Fig. 1). In one of the peripheral boxes (except the leftmost box ${ }^{1}$ ), a green target symbol ("X" or "="; $3.67^{\circ}$ tall) could appear for $50 \mathrm{~ms}$. White distractor symbols appeared in the other peripheral boxes. The participant's goal was to report the identity of the

\footnotetext{
${ }^{1}$ This was an unintentional modification to the paradigm as a result of a programming error. This modification would have essentially made capture more difficult to find because it made the cue less predictive, yet we find robust capture. When the cue appeared at the leftmost box, the target location could only be at the top, bottom, or right boxes.
}

target as quickly and accurately as possible (within a 1,500ms response window) while keeping his or her gaze on the central box and ignoring a cue that preceded the target and did not predict the target location. If a particular type of cue captures attention, on valid trials (trials on which the cue and target coincide spatially-typically, $25 \%$ of trials), performance is expected to be better relative to invalid trials (i.e., nonmatching locations between cue and target-typically, $75 \%$ of trials).

Participants completed a color cue and onset cue block of the task. In the color cue block, participants saw a green cue around one of the four outer boxes (four colored circles with a $0.46^{\circ}$ radius, one located on each side of the box) and a white cue around the other three boxes prior to the target appearing (180 trials). In this condition, it was expected that attention would be captured by the cue that matches the attention set of the participant (searching for green target, green cue appears before). In the onset cue block of the task, participants saw a white cue around one of the four outer boxes, and no cue around the other three boxes (180 trials). In this condition, it was expected that the cue would be more easily ignored because it does not match the attention set of the participant. Block order was determined randomly at the beginning of the experiment for each participant. Within-block target identity and cue validity was determined randomly for each trial.

Contingent blink paradigm (based on Folk, Leber, \& Egeth, 2002) For this task (created using E-Prime; Psychology Software Tools, Pittsburgh, PA), following the presentation of a fixation cross for $500 \mathrm{~ms}$, participants saw a stream of 15 letters $\left(1.3^{\circ}\right.$ height, $1.2^{\circ}$ width) rapidly presented centrally in the display (each letter for $42 \mathrm{~ms}$, followed by a 42-ms blank) on a black background (see Fig. 2). The goal was to identify and report the green letter presented in the stream among different-colored distractor letters. This green letter occurred equally often in stream positions 8 through 12 . On distractor-present trials ( $75 \%$ of trials), four pound signs $(\#)$ appeared around the centrally presented letter $\left(5.2^{\circ}\right.$ above, below, left, and right), varying in color for each of the three possible distractor conditions. These distractor objects appeared either simultaneously with the target (i.e., lag 0 ), or one to three positions (i.e., lags 1 through 3 ) before the target in the stream. In one condition, all of the pound signs were gray ( $25 \%$ of trials). In the other two conditions, one pound sign (randomly selected) was either red or green, and the others were gray ( $25 \%$ of trials for each color). In the distractor-present condition in which the distractor color matched the target color, it was expected that accuracy would be lower than when the distractor color did not match the target color, or when it was absent altogether. Trial order, distractor type, and distractor lag position were determined through randomization for all 165 trials. 


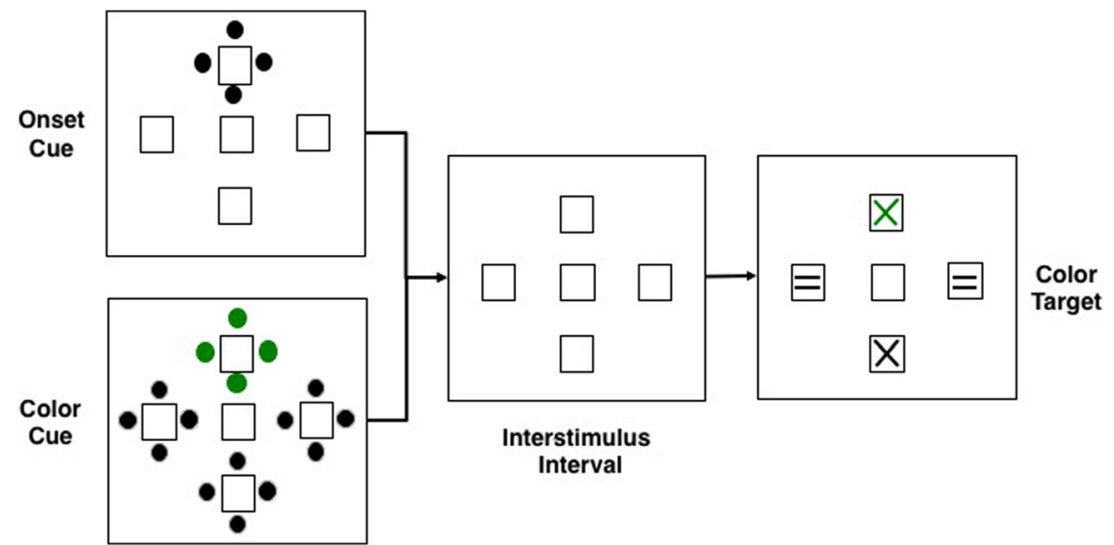

Fig. 1 Depiction of the contingent capture paradigm used in the present study (adapted from Folk, Remington, \& Johnston, 1992). Participants reported the identity of the green symbol. (Color figure online)

\section{Bottom-up tasks}

Additional singleton paradigm (based on Theeuwes, 1992) For this task (created using OpenSesame; Mathôt, Schreij, \& Theeuwes, 2012), participants searched a display with either three or seven objects on the screen, laid out along an invisible circle with a radius of $3.4^{\circ}$, on a black background (see Fig. 3 ). The display contained three objects $50 \%$ of the time and seven objects $50 \%$ of the time. One of the objects was a green circle $\left(1.4^{\circ}\right.$ diameter $)$, whereas the others were square $\left(1.4^{\circ}\right.$ on each side). These square nontarget objects contained lines $\left(0.5^{\circ}\right.$ length $)$ as well, but they were tilted $22.5^{\circ}$ away from being either horizontal or vertical. On distractor-present trials (50\% of trials; 72 trials), one red square (i.e. color singleton) appeared on-screen, whereas the other distractors were green squares. On distractor-absent trials (50\% of trials; 72 trials), all distractor items were green squares. The goal in this task was to report the orientation of a line $\left(0.5^{\circ}\right.$ in length, either horizontal or vertical) within the green circle while maintaining gaze on the central fixation point. Feedback was administered after each response was made by changing the fixation dot to green if the response was correct and red if it was incorrect. It was expected that responses would be slowed when a red distractor item appeared on-screen with the green target item. Observers also participated for 144 trials in a condition where all the nontarget items were red circles, and on distractor-present trials, the distractor was a shape singleton (i.e., red square). These trials were not analyzed (capture is typically not observed in these cases). Trial order, set size, and distractor presence were all determined through randomization.

Irrelevant singleton paradigm (based on Jonides \& Yantis, 1988; Yantis \& Jonides, 1984) For this task (using PsychoPy), participants searched a display for a target letter (either $H$ or $U$ ) within a search array of objects laid out along an imaginary circle $\left(4.5^{\circ}\right.$ radius $)$, with 10 possible evenly spaced locations (see Fig. 4). Participants were instructed to maintain their gaze on the fixation dot in the center of the screen throughout the task. The task began with either two or six figure-eight premasks $\left(1.9^{\circ}\right.$ length and height) appearing on screen for $1,000 \mathrm{~ms}$. Because there were fewer masks than objects in the final search display, this created a visual onset, resulting

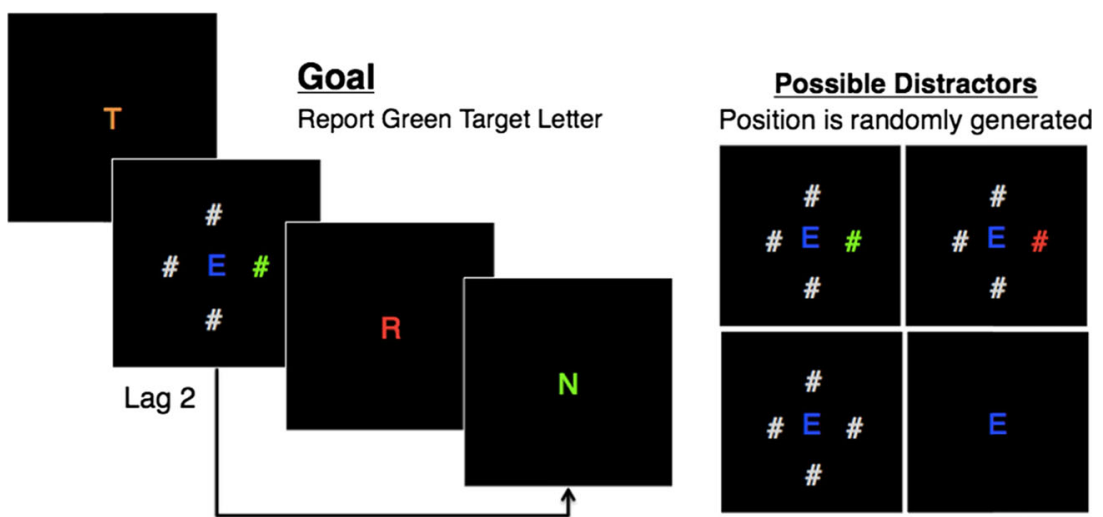

Fig. 2 Depiction of the contingent blink paradigm, using many different-colored nontarget items (adapted from Folk, Leber, \& Egeth, 2002). Participants reported the identity of the green letter. (Color figure online) 


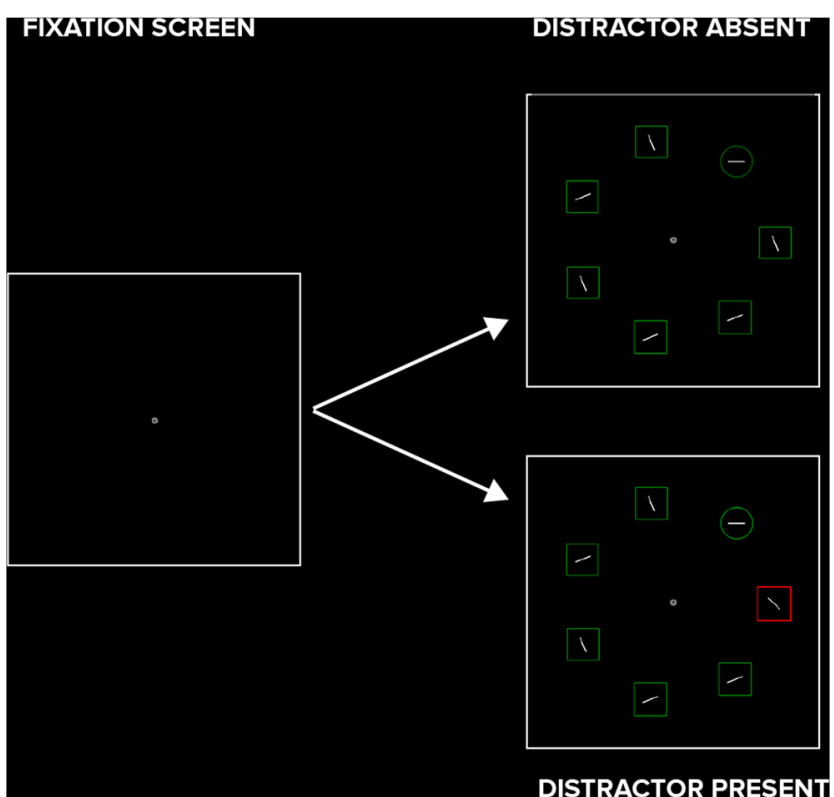

Fig. 3 Depiction of the additional singleton paradigm (adapted from Theeuwes, 1992). Participants reported the orientation of the line within the green circle. (Color figure online)

in a search display of either three or seven objects. There were 90 trials for which there were three objects in the display and 90 trials for which there were seven objects in the display. Participants were told that there was no advantage to prioritizing the onset item (at each set size, the target could be the onset $20 \%$ of the time, meaning that the target was a nononset item more often than not). After the premasks were drawn, the subsequent screen showed the search array of letters, by removing segments of the figure-eight objects. The final search array contained either three or seven objects, one of which was the target $(H$ or $U$ ) whereas the rest were distractors $(A, C, E, F, L, P$, or $S)$. It was expected that when the onset was the target, reaction times would be

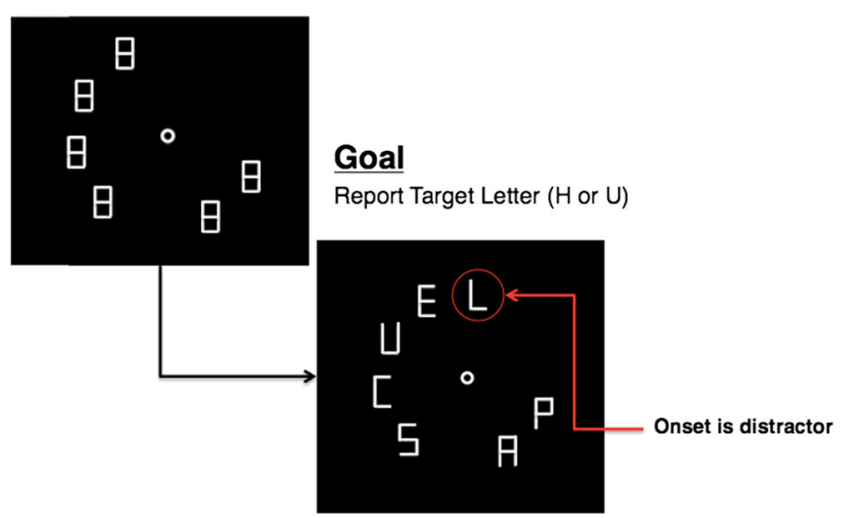

Fig. 4 Depiction of the irrelevant singleton paradigm (Yantis \& Jonides, 1984). In this example, the onset is a distractor. Participants searched for an $\mathrm{H}$ or $\mathrm{U}$ relatively invariant to set size because the target would be one of the first items to be searched. When the onset was the distractor, it was expected that reaction times would increase sharply with additional items added to the display because each item would need to be searched serially before the target was found. As is tradition with this paradigm, capture was assessed by the search slope when the target was the onset item relative to when it was not the onset item. Trial order, set size, and onset identity (i.e., distractor or target) was determined through randomization.

\section{Procedure}

Upon entering the lab and giving consent, participants completed all four tasks listed in the Materials section. Task order was counterbalanced in this experiment to control for a possible effect of task order. For each task, experimenters read instructions directly from the screen, word for word, emphasizing the idea that participants should keep their gaze at the center of the screen and respond without making eye movements.

\section{Capture results}

First, we present the capture results of Experiment 1. Analyses focused on whether or not classic attention capture effects would be observed.

\section{Trial exclusion}

In cases in which response times were to be analyzed, only response times from accurate trials were considered. In cases in which a participant's task performance, as measured through accuracy, was less than three standard deviations below the mean, that specific task's capture scores were dropped from subsequent correlational and principal components analyses (PCA). This led to a loss of five participants in the contingent cuing paradigm, six participants in the contingent blink paradigm, five participants in the additional singleton paradigm, and 10 participants in the irrelevant singleton paradigm.

\section{Replications of classic top-down capture effects}

Contingent cuing paradigm (Folk, Remington, \& Johnston, 1992) Observers searched for a target of a specific color (green), and an irrelevant cue preceded the target that was either consistent (green color cue) or inconsistent (onset cue) with the target-defining feature. Reaction times from the contingent cuing paradigm were entered into an ANOVA with cue type (color or onset) and validity (valid or invalid) as within- 
subjects factors. Critically, a significant cue type and cue validity interaction was observed, $F(1,145)=324.93, p<.001$. Replicating Folk and colleagues (1992), the irrelevant cue captured attention (as evidenced by a validity effect) only when it was consistent with the observers' target (see Fig. 5, left panel). This contingent capture resulted in slower reaction times when the target-consistent color cue occurred in an invalidly cued location compared to a valid target location, $t(145)=21.13, p<.001$ ( $M$ diff $=-66 \mathrm{~ms}, S D=38 \mathrm{~ms})$. The onset cue did not capture attention, $t(145)=.09, p=$ $.93(M$ diff $=1 \mathrm{~ms}, S D=27 \mathrm{~ms})$. In sum, this strong replication of Folk et al. (1992) suggests that top-down factors are critical in determining where observers allocate their attention. Capture occurred only when cues matched the participant's attention set, supporting the notion of contingent capture.

Contingent blink paradigm (Folk, Leber, \& Egeth, 2002) If observers' attention sets determine the extent to which an irrelevant distractor captures attention, we would expect observers' accuracy to be worse when the irrelevant distractor matches the observers' attention set, as in these cases the distractor would pull attention away from the target location. An ANOVA was performed with distractor type (green, gray, red, or none) and lag (lag 0, 1, 2, 3) as within-participants factors. Results of the ANOVA revealed a significant interaction between distractor type and distractor lag, $F(9,1305)=$ 82.77, $p<.001$. As can be seen from Fig. 6, there was little difference between when the peripheral distractors were not consistent with the participants' attention set (gray, red) or were absent, so these conditions were collapsed in subsequent analyses. Accuracy remained near ceiling for all conditions at the earliest lag, with the green distractor not significantly impairing performance compared to the other distractor conditions, $t(145)=-1.49, p=.14(M$ diff $=-1 \%, S D=7 \%)$. For all the other lags, however, when the green distractor was present, accuracy was significantly impaired compared to other distractors: lag $1, t(145)=-10.33, p<.001(M$ diff $=-15 \%$, $S D=18 \%)$; lag 2, $t(145)=-17.24, p<.001(M$ diff $=-32 \%$, $S D=22 \%)$; lag $3 t(145)=-14.82, p<.001(M$ diff $=-28 \%$, $S D=23 \%$ ). In sum, this strong replication of Folk et al. (2002) further suggests that top-down factors are critical in determining where observers allocate their attention. Capture only occurred when the peripheral distractor matched the participant's attention set.

\section{Replications of classic bottom-up capture effects}

Additional singleton paradigm (Theeuwes, 1992) Observers in the additional singleton paradigm were asked to report the orientation of a line within a target shape (i.e., green circle). On some trials, a unique distractor would be present (i.e., a red square). If observers' attention is captured by unique features in the display, regardless of their current goals, we would expect participants' reaction time to be slower on trials in which the distractor is present compared to when the distractor is absent. Participants' reaction times were entered into an ANOVA with distractor presence (present and absent) and set size ( 3 and 7) as within-subjects factors. Replicating Theeuwes (1992), a main effect of distractor presence was found, $F(1,145)=4.90, p=.03$. Observers were slower to identify their target on trials in which the salient distractor was present compared to when the distractor was absent ( $M$ diff $=7 \mathrm{~ms}, S D=39 \mathrm{~ms}$ ). This effect appeared to be primarily driven by increased capture in the large set-size condition, as evident by the significant distractor presence and set-size interaction, $F(1,145)=36.42, p<.001$. Observers were approximately $23 \mathrm{~ms}(S D=56 \mathrm{~ms})$ slower to locate their target in the large set-size condition when the salient distractor was present compared to when it was absent, $t(145)=4.96, p$ $<.001$ (see Fig. 7, left panel). However, this slowing was not evident in the small set-size condition, as observers were approximately $9 \mathrm{~ms}(S D=44 \mathrm{~ms})$ faster to locate their target when the salient distractor was present compared to when it was absent, $t(145)=-2.35, p=.02$. In sum, ${ }^{2}$ the color singleton captured attention even though participants were told it was irrelevant and that they should only attend to the item of a unique shape. However, this did not represent a complete replication, given the unusual pattern observed in the small set-size condition. Thus it was decided to use only the set-size seven condition in subsequent correlational analyses.

Irrelevant singleton paradigm (Yantis \& Jonides, 1984) Observers were asked to report the identity of a target letter as either $H$ or $U$. An onset appeared on each trial, and the onset could either be the target item or a distractor. If the onset captures attention, search slopes should be flatter when the onset is the target compared to the distractor. Participants' reaction times were entered into an ANOVA with onset type (target and distractor) and set size (3 and 7) as within-subjects factors. Replicating Yantis and Jonides (1984), a significant interaction between onset type and set size was observed, $F(1$, $145)=131.7, p<.001$ (see Fig. 8, left panel). Although both onset conditions appear to have significant search slopes - as is evident by one-sample $t$ tests comparing the search slopes to zero: onset distractor, $t(145)=34.02, p<.001, M=46 \mathrm{~ms} /$ item, $S D=16 \mathrm{~ms} /$ item ; onset target, $t(145)=11.07, p<.001$, $M=22 \mathrm{~ms} /$ item, $S D=24 \mathrm{~ms} /$ item-this interaction was

\footnotetext{
${ }^{2}$ The original Theeuwes study from 1992 did not include a set-size three condition. This modification to the paradigm was done in order to make the task more comparable to the irrelevant singleton paradigm. The pattern of no capture, or faster RTs, when the additional singleton was present is unusual. This may be explained by the red distractor being less unique (and perhaps easier to ignore) with only two green items in the display. Gestalt grouping principles may have helped participants isolate their search to only the two relevant items.
} 


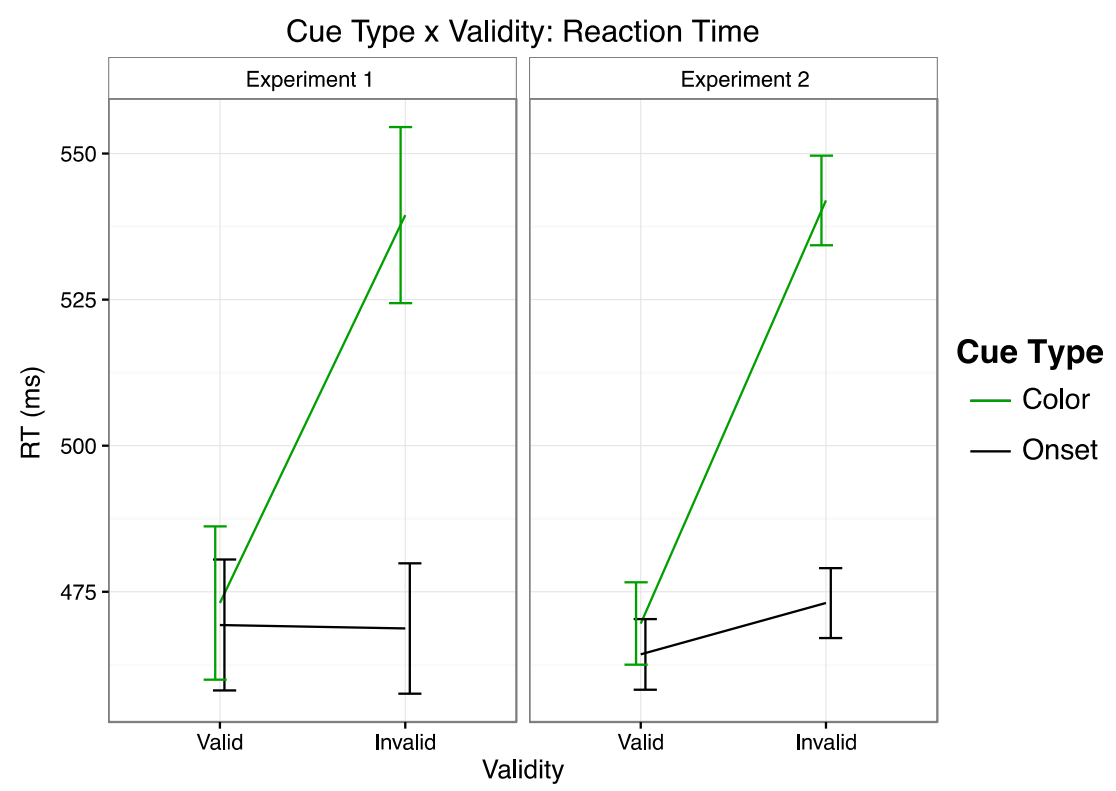

Fig. 5 Depiction of the replication results for the contingent capture paradigm (Experiments 1 and 2)

driven by a significantly steeper search slope in the onset distractor condition compared to the onset target condition, $t(145)=11.48, p<.001$ ( $M$ diff $=24 \mathrm{~ms} /$ item,$S D=25 \mathrm{~ms} /$ item). This suggests that attention was likely allocated to onset item early in the search process, a sign of capture. In sum, this replication of Yantis and Jonides (1984) further demonstrates that bottom-up factors are potentially critical in determining where observers allocate their attention. Despite providing little predictive value, the onset item was prioritized.

\section{Experiment 2}

The results of Experiment 1 closely matched findings in the literature. However, there were some unusual aspects of the reported data as well. This included no capture observed in the small set-size condition of the additional singleton paradigm. This small set size is atypical for this paradigm. Also, the search slope when the target was an onset in the irrelevant singleton paradigm was not flat, as has often been reported

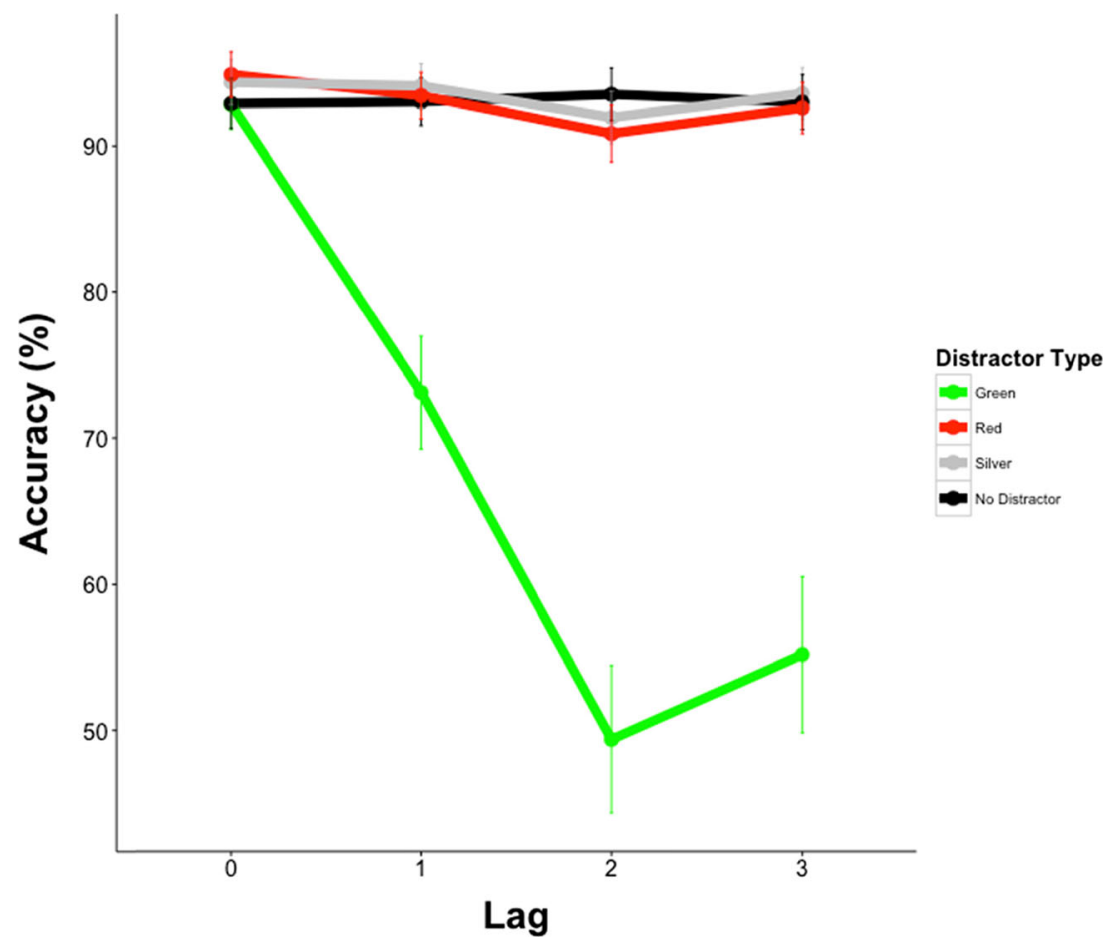

Fig. 6 Depiction of the replication results for the contingent blink paradigm (Experiment 1 only) 
Set Size x Distractor: Reaction Time

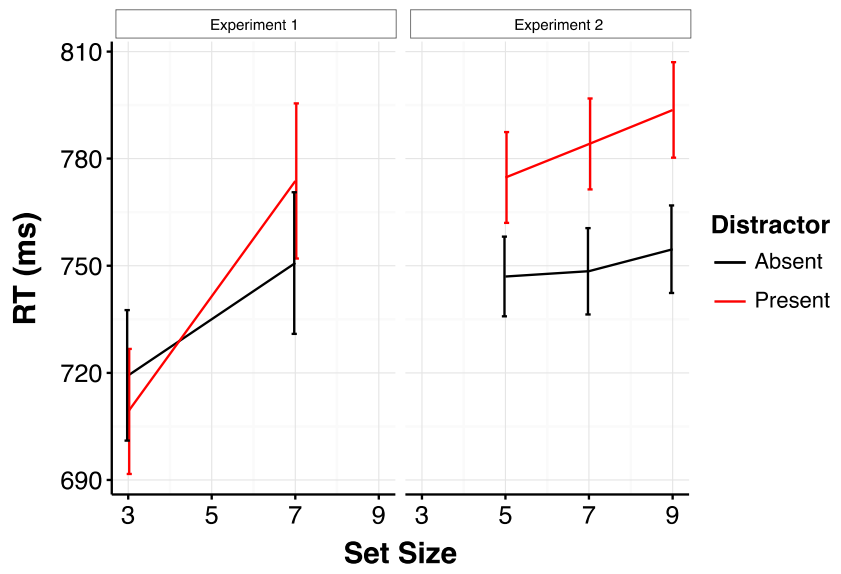

Fig. 7 Depiction of the replication results for the additional singleton paradigm (Experiments 1 and 2)

in the literature. This may be due to our modification of the paradigm, in which the $20 \%$ of trials of each set size featured the target as the onset, making the onset antipredictive in the small set-size condition, and slightly predictive in the large set-size condition. We decided to collect more data from a separate group of participants addressing these issues. We also used this opportunity to correct the previously mentioned programming issue in the contingent cuing paradigm and increase the number of trials for some of the paradigms. To increase the number of trials and maintain an experimental session length that would minimize fatigue, the contingent blink paradigm was not tested again.

\section{Method}

\section{Participants}

One hundred fourteen undergraduate students with selfreported normal color vision participated in this study for

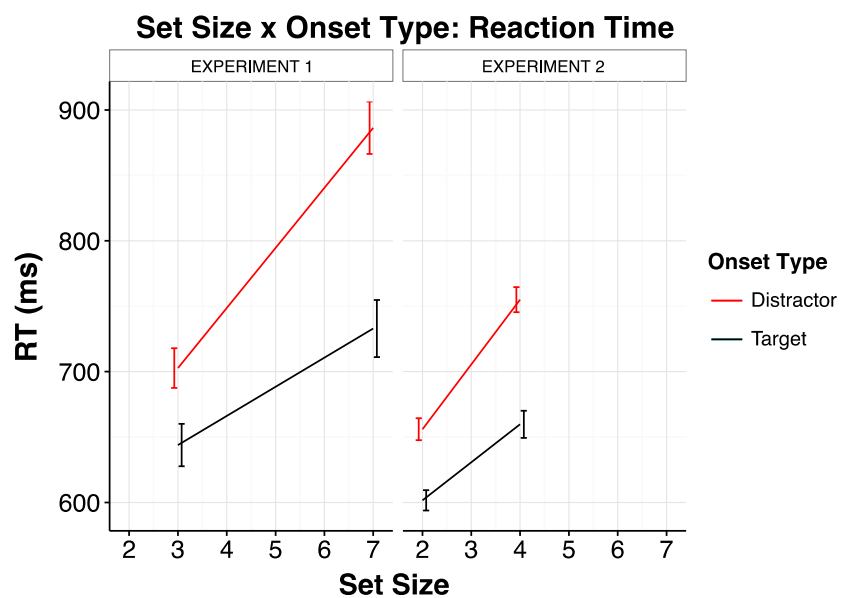

Fig. 8 Depiction of the replication results for the irrelevant singleton paradigm (Experiments 1 and 2) course credit. This sample had an average age of 20 years $(S D=1.46)$.

\section{Materials}

Same as Experiment 1. Each task used in this study is described in more detail below.

\section{Overview}

For the purposes of equating all four tasks in Experiment 1, certain decisions were made, such as using set sizes 3 and 7 for the additional singleton paradigm and irrelevant singleton paradigm. In addition, the probability with which the target occurred as an onset was $20 \%$ across both display size conditions in the irrelevant singleton paradigm, resulting in the target being antipredictive at set size 3 and predictive at set size 7. For Experiment 2, these tasks were modified to better match the original experiments (Theeuwes, 1992, 1994; Jonides \& Yantis, 1988; Yantis \& Jonides, 1984), in both trial probability (i.e., irrelevant singleton paradigm) and trial blocking (i.e., additional singleton paradigm).

\section{Top-down tasks}

Contingent cuing paradigm (based on Folk, Remington, \& Johnston, 1992) The methods used for this experiment did not change from Experiment 1, except for ensuring that the target also appeared in the left-most box.

\section{Bottom-up tasks}

Additional singleton paradigm (based on Theeuwes, 1992) The set sizes tested were made more similar to previous studies, and distractor-present versus distractor-absent conditions were blocked, as has been done previously (Theeuwes, 1992). The display contained five objects one-third of the time, seven objects one-third of the time, and nine objects one-third of the time, for a total of 300 nonpractice trials. The red color singleton distractor was present on $50 \%$ of all trials. Distractor presence was blocked and counterbalanced. All other aspects of the task were identical to the same task in Experiment 1. Trial order, set size, and distractor presence were all determined through randomization.

Irrelevant singleton paradigm (based on Jonides \& Yantis, 1988; Yantis \& Jonides, 1984) For this task (using PsychoPy), participants searched a display for a target letter (either $H$ or $U$ ) within a search array of objects laid out along an imaginary circle $\left(9^{\circ}\right.$ diameter), with 10 possible evenly spaced locations. Set size was changed to two and four items. There were 100 trials for which there were two objects in the 
display, and 100 trials for which there were four objects in the display. In the small set-size condition (set size 2), the onset was the target $50 \%$ of the time. In the large set-size condition (set size 4 ), the onset was the target $25 \%$ of time. This made the onset nonpredictive of the target location in both set-size conditions. All other aspects of the task were identical to the same paradigm in Experiment 1. Trial order, set size, and onset identity (i.e., distractor or target) was determined through randomization.

\section{Procedure}

Upon entering the lab and giving consent, participants completed all three tasks. Task order was counterbalanced in this experiment to control for a possible effect of task order. For each task, experimenters read instructions directly from the screen, word for word, emphasizing the idea that participants should keep their gaze at the center of the screen and respond without making eye movements.

\section{Results}

\section{Trial exclusion}

Because of experimenter or computer error, 10 participants in total did not have complete datasets for all three tasks. All subsequent analyses begin with a dataset containing 104 participants with all three tasks completed. In cases in which response times were to be analyzed, only response times from accurate trials were considered. In cases in which a participant's task performance, as measured through accuracy, was less than three standard deviations below the mean, that specific task's capture scores were dropped from subsequent correlational and PCA analyses. This led to a loss of five participants in the contingent cuing paradigm, seven participants in the additional singleton paradigm, and four participants in the irrelevant singleton paradigm.

\section{Replications of classic top-down capture effects}

\section{Contingent cuing paradigm (Folk, Remington, \&} Johnston, 1992) Reaction times from the contingent cuing paradigm were entered into an ANOVA with cue type (color or onset) and validity (valid or invalid) as withinsubjects factors. Recall that participants were searching for a target of a unique color (green). Replicating Experiment 1, a significant cue type and cue validity interaction was revealed, $F(1,103)=236.78, p<.001$. The cue captured attention (as evidenced by a validity effect) when it was consistent with the observers' target. As is evident in Fig. 5 (right panel), this contingent capture resulted in slower reaction times when the targetconsistent color cue occurred in an invalidly cued location compared to a valid target location, $t(103)=$ 19.92, $p<.001$ ( $M$ diff $=-72 \mathrm{~ms}, S D=37 \mathrm{~ms}$ ). The onset cue produced a validity effect, but it was of a substantially smaller magnitude, $t(103)=-3.75, p<.001$ ( $M$ diff $=-9 \mathrm{~ms}, S D=24 \mathrm{~ms}$ ).

\section{Replications of classic bottom-up capture effects}

Additional singleton paradigm (Theeuwes, 1992) Participants' reaction times were entered into an ANOVA with distractor presence (present and absent) and set size $(5,7,9)$ as within-subjects factors. Replicating Experiment 1, a main effect of distractor presence was found, $F(1,103)=36.948, p<.001$. Observers were slower to identify their target on trials in which the salient distractor was present compared to when the distractor was absent $(M$ diff $=34 \mathrm{~ms}, S D=57 \mathrm{~ms})$. A main effect of set size was also found, $F(2,206)=7.361, p=.001$, where reaction time increased linearly as a function of set size (set size 5: $M=760.9 \mathrm{~ms}, S D=118 \mathrm{~ms}$; set size 7: $M=$ $766.3 \mathrm{~ms}, S D=122 \mathrm{~ms}$; set size 9: $M=774 \mathrm{~ms}, S D=$ $126 \mathrm{~ms})$. No interaction was observed between set size and distractor presence, $F(2,206)=2.063, p=.130$. Figure 7 (right panel) depicts these results.

Irrelevant singleton paradigm (Yantis \& Jonides, 1984) Replicating Yantis and Jonides (1984), a significant interaction between onset type and set size was observed, $F(1,103)=$ 40.597, $p<.001$ (see Fig. 8, right panel). Although both onset conditions appear to have significant search slopes-as evident by one-sample $t$ tests comparing the search slopes to zero: onset distractor, $t(103)=22.152, p<$ $.001, M=50 \mathrm{~ms} /$ item, $S D=23 \mathrm{~ms} /$ item; onset target, $t(103)=9.680, p<.001, M=29 \mathrm{~ms} /$ item,$S D=30 \mathrm{~ms} /$ item - this interaction was driven by a significantly steeper search slope in the onset distractor condition compared to the onset target condition, $t(103)=6.37, p<.001(M$ diff $=20 \mathrm{~ms} /$ item,$S D=33 \mathrm{~ms} /$ item $)$. Despite providing no predictive value, the onset item was prioritized. Once again, search slopes when the onset was the target were not flat. However, this pattern is not inconsistent with other reports in the literature (Boot et al., 2005; Franconeri \& Simons, 2003).

\section{Relationship between capture meausures}

Having demonstrated capture effects across several paradigms used in support of top-down and bottom-up capture, we next turn to the correlations between measures in Experiment 1, and in Experiment 2. 
Table 1 Operational definitions of final capture measures used

Capture score Operational definition

Top-down measures

Contingent cuing

(green distractor invalid inefficiency - green distractor valid inefficiency) / green distractor valid inefficiency

Contingent blink

(green distractor lag 2 - red distractor lag 2) / red distractor lag 2

Bottom-up measures

Irrelevant singleton

Additional singleton

onset distractor inefficiency search slope - onset target inefficiency search slope

Onset cuing

(distractor present set size 7 inefficiency - distractor absent set size 7 inefficiency) / distractor absent set size 7 inefficiency

Color singleton blink (onset distractor invalid inefficiency - onset distractor valid inefficiency) / onset distractor valid inefficiency (red distractor lag 2 - silver distractor lag 2) / silver distractor lag 2

Note. All RT measures were converted to inefficiency scores to reflect both response time and accuracy (RT/proportion accuracy). Because of the nature of the task (nonspeeded), scores derived from the contingent blink paradigm only considered accuracy

\section{Capture measures: Defined}

It was necessary to derive an index of the magnitude of attentional capture by task-irrelevant information (e.g., observing a red square) while working toward a top-down goal (e.g., searching for a green circle) in order to evaluate the relationship between measures from different paradigms. This index, hereafter referred to as capture scores, was computed for each task, yielding six capture scores (two top-down measures, four bottom-up measures) for Experiment 1 and four capture scores (one top-down, three bottom-up) for Experiment 2. Two of the bottom-up capture scores were uniquely derived from topdown tasks (i.e., contingent cuing: onset capture score,

Table 2 Reliability of originally defined capture scores for Experiments 1 and 2

\begin{tabular}{lll}
\hline $\begin{array}{l}\text { Experiment } 1 \\
\text { Capture scores }\end{array}$ & Split-half reliability & Parity reliability \\
Top-down measures & & \\
$\quad$ Contingent cuing & $.34^{* *}$ & $.23^{* *}$ \\
Contingent blink & $.45^{* *}$ & $.56^{* *}$ \\
Bottom-up measures & & \\
Irrelevant singleton & .12 & .13 \\
Additional singleton & .09 & .02 \\
Onset cuing & $.20^{*}$ & $.29^{* *}$ \\
Color singleton blink & .02 & .02 \\
Experiment 2 & & \\
Top-down measures & & $.43^{* *}$ \\
Contingent cuing & $.48^{* *}$ & \\
Bottom-up measures & & .17 \\
Irrelevant singleton & .10 & $.50^{* *}$ \\
Additional singleton & .13 & $.19^{*}$ \\
Onset cuing & $.28^{* *}$ &
\end{tabular}

Note. Split-half and parity reliability were compared

*indicates significant at the 0.05 level. $* *$ indicates significant at the 0.01 level contingent blink: color singleton score). The motivation for this was to extract additional information from these tasks in which unique attributes might capture some participants' attention, so we evaluated these tasks for instances in which unique distraction was present compared to absent. For example, in the contingent blink paradigm, this score was calculated as the difference in accuracy at lag 2-where the largest capture effect was observed (see Fig. 6) - when the red distractor appeared relative to the gray distractor (because participants were searching for green, this would represent a bottom-up effect). This approach of creating additional bottom-up measures was validated because the distribution of capture scores (presented later) indicated that a subset of

Table 3 Reliability of efficiency-based capture scores

$\begin{array}{lll}\begin{array}{l}\text { Experiment } 1 \\ \text { Capture efficiency scores }\end{array} & \text { Split-half reliability } & \text { Parity reliability } \\ \begin{array}{l}\text { Top-down measures } \\ \text { Contingent cuing }\end{array} & .42^{* *} & .32^{* *} \\ \text { Bottom-up measures } & & \\ \quad \text { Irrelevant singleton } & .04 & .12 \\ \text { Additional singleton } & .06 & -.03 \\ \text { Onset cuing } & .27^{* *} & .04 \\ \text { Experiment 2 } & & \\ \text { Top-down measures } & & \\ \text { Contingent cuing } & .47^{* *} & .40^{* *} \\ \text { Bottom-up measures } & & .20^{*} \\ \text { Irrelevant singleton } & .25^{*} & .48^{* *} \\ \text { Additional singleton } & .23^{*} & -.05 \\ \text { Onset cuing } & .27^{* *} & \end{array}$

Note. Split-half and parity reliability were compared. Efficiency scores were not calculated for the contingent blink paradigm, because there was no index of reaction time for that task (participants are pushed to be accurate, not fast)

*indicates significant at the 0.05 level. **indicates significant at the 0.01 level. 


\section{EXPERIMENT 1 \\ Correlations between Top-Down \& Bottom-Up Capture Measures}

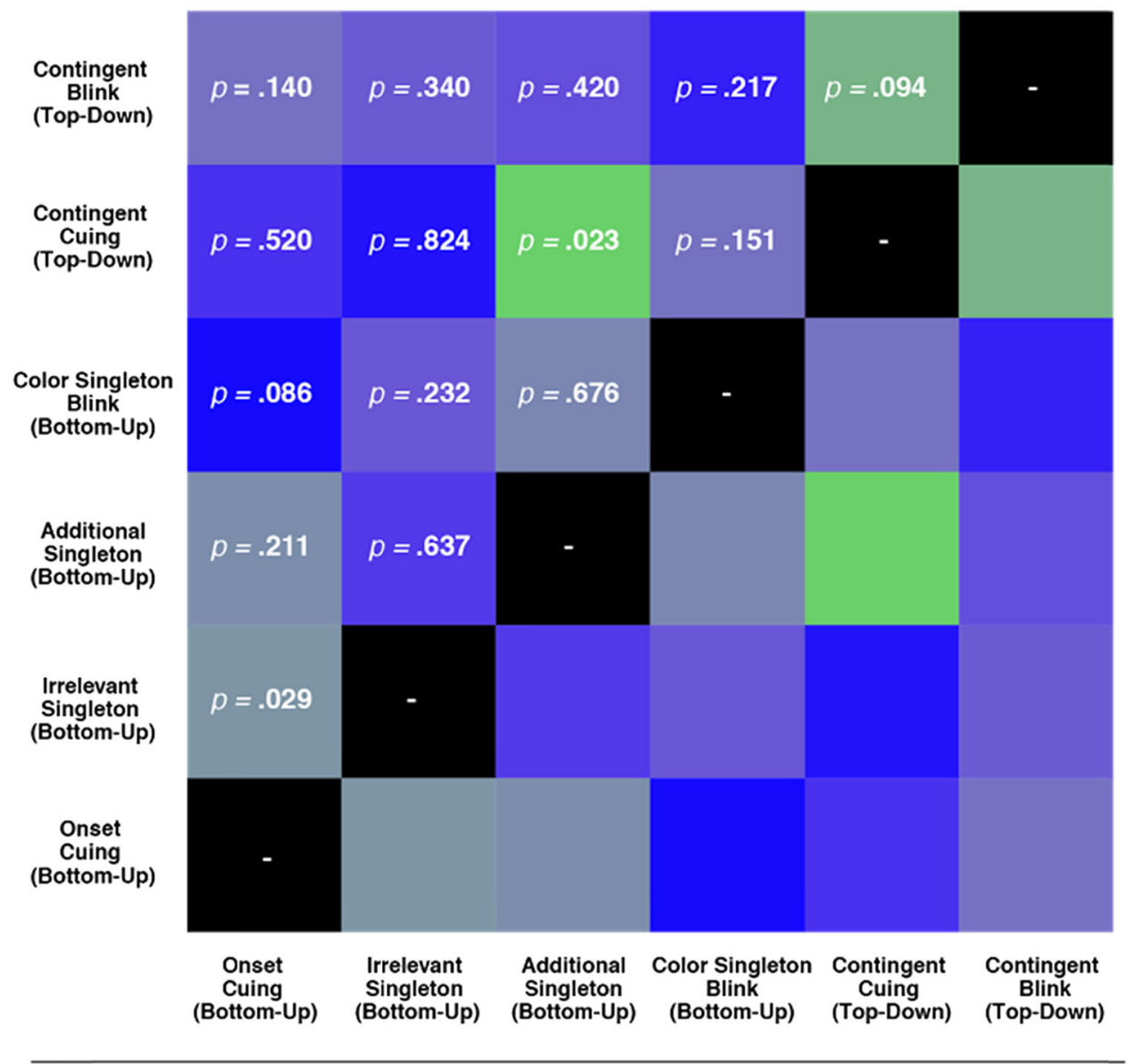

\section{Correlation}

Fig. 9 Correlation matrix heat map for the efficiency-based capture scores in Experiment 1. Absolute value of correlations are depicted. Blue indicates weaker correlations, and red indicates stronger correlations. (Color figure online)

participants demonstrated bottom-up capture effects in these conditions in measures typically used to study contingent capture (see Table 1 for operational definitions of each measure).

To maximize the amount of information captured by our capture scores, we focused on inefficiency-based scores that take into account both the speed and accuracy of performance for a condition. Inefficiency-based capture scores involve the division of reaction time by accuracy in each condition, to produce scores that speak to both speed and accuracy (Townsend \& Ashby, 1983). It should be noted, however, that scores that did and did not include accuracy were highly correlated for each paradigm $(>.70)$. For paradigms in which responses are typically unspeeded, capture scores were only computed using accuracy (i.e., contingent blink paradigm). All analyses without inefficiency-based measures are included in a supplement.

\section{Reliability of capture scores}

We explored the reliability of capture scores derived only from response times (see Table 2) and also from inefficiency scores (see Table 3). Focusing on Experiment 2, which includes more trials and was a more direct replication of previous capture studies, there was more evidence for reliability when using inefficiency-based scores (i.e., a greater number of significant correlations with respect to split-half and parity reliability calculations). In particular, for the irrelevant singleton paradigm, no significant reliability was observed in Experiment 2 when only scores based on response times were used, whereas significant reliability was observed when inefficiency scores were used. However, reliability in general was very low. It is important to note however, that these estimates of reliability 


\section{EXPERIMENT 2}

\section{Correlations between Top-Down \& Bottom-Up Capture Measures}

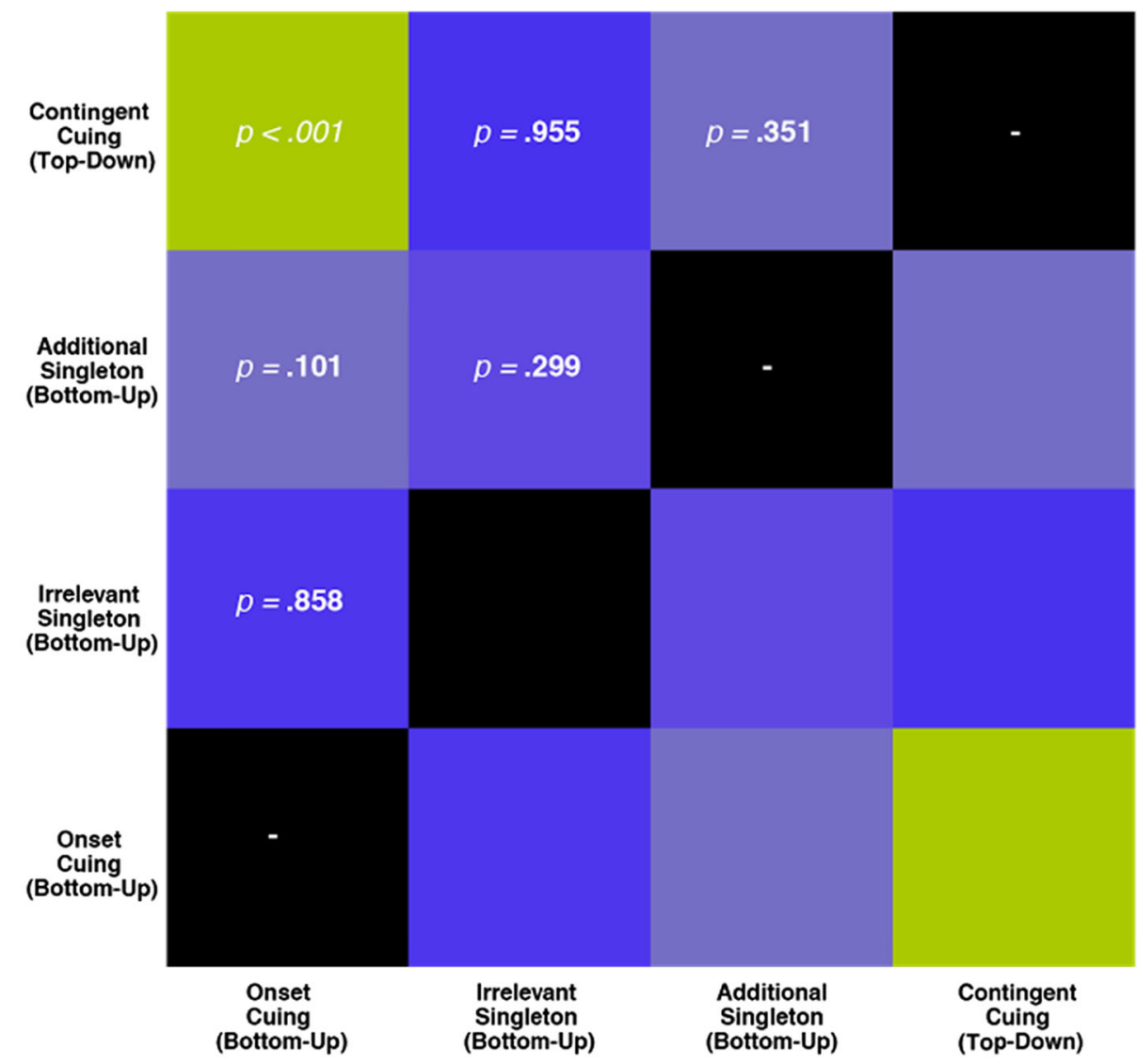

\section{Correlation}

$0.0 \quad 0.1$

0.1

0.2

0.3

Fig. 10 Correlation matrix heat map for the efficiency-based capture scores in Experiment 2. Absolute value of correlations are depicted. Blue indicates weaker correlations, and red indicates stronger correlations. (Color figure online)

may be underestimating the true reliability of these measures. For example, in the irrelevant singleton paradigm in Experiment 1, the target was the onset $20 \%$ of the time in the set size 7 condition, resulting in 18 trials. To measure the reliability of this condition using the split-half technique would result in only nine trials per half. This, however, was less of a problem in Experiment 2 with smaller set sizes, and thus a greater number of onset target trials.

\section{Experiment 1 and 2 correlations}

If capture represents a unitary phenomenon, there should be a high correlation between capture in these paradigms, but if there are different types of capture (top-down, bottom-up), some attention capture effects should be correlated and some should not. Figures 9 and 10 represent heat maps depicting the pattern of correlations between capture scores. Notably, there are few instances of high correlations. One might expect bottom-up measures to be strongly correlated with each other, top-down measures to be strongly correlated with each other, and top-down measures to be weakly correlated with bottomup measures, if at all. For example, one would expect capture scores in the additional singleton paradigm to be strongly related to capture scores in the irrelevant singleton paradigm since both have been purported to measure bottom-up, stimulus driven capture. Instead, it was found that bottom-up measures were not significantly correlated with each other in Experiments 1 and 2 (additional singleton and irrelevant singleton: Experiment 1, $r=-.041, p=.637$; Experiment 2, $r=$ $.108, p=.299$ ). In Experiment 1, the two top-down measures 
Table 4 PCA results for inefficiency-based capture scores (varimax rotated solution)

Experiment 1

\begin{tabular}{llll} 
& Component & & \\
& 1 & 2 & 3 \\
Irrelevant singleton (BU) & $\mathbf{. 7 4 8}$ & .020 & -.195 \\
Onset cuing (BU) & $\mathbf{. 7 2 2}$ & -.095 & .203 \\
Contingent blink (TD) & -.089 & $\mathbf{. 8 0 2}$ & .254 \\
Contingent cuing (TD) & -.129 & $\mathbf{- . 6 0 8}$ & .533 \\
Color singleton blink (BU) & -.383 & .405 & -.130 \\
Additional singleton (BU) & .085 & .104 & $\mathbf{. 8 6 3}$ \\
Experiment 2 & & & \\
& Component & & \\
Contingent cuing (TD) & 1 & 2 & \\
Onset cuing (BU) & $\mathbf{. 8 2 7}$ & .213 & \\
Irrelevant singleton (BU) & $\mathbf{. 8 0 7}$ & -.224 & \\
Additional singleton (BU) & .092 & $\mathbf{. 8 2 7}$ & \\
\hline
\end{tabular}

Note. $\mathrm{BU}=$ bottom-up, $\mathrm{TD}=$ top-down Factor loadings above .6 are in bold face for ease of viewing.

of capture were also not correlated (contingent cuing: color cue measure and contingent blink top-down measure, $r=$ $0.144, p=.094)$. A correlation that was quite surprising in Experiment 1 was between one top-down measure (contingent cuing: color cue) and a bottom-up measure (additional singleton measure; $r=.194, p=.023$ ). These two measures have been used to support opposing views of the nature of capture but, to some (small) extent, appear to be measuring the same thing. Note that this correlation was not significant in Experiment $2(r=-.098, p=.351)$. Another correlation that was quite surprising in Experiment 2 was between the topdown and bottom-up measures calculated from the contingent cuing paradigm $(r=.344, p<.001)$. In Experiment 2 , the degree of capture observed in the contingent cuing paradigm by color singleton cues was positively correlated with capture by onsets. Note again, that this was not replicated in Experiment 1.

\section{PCA results}

Initially, a confirmatory factor analysis was intended to explore one- and two-factor solutions, but we experienced problems with model identifiability. Thus, a data-driven approach was taken in order to best understand the underlying structure of the capture scores derived from top-down and bottom-up paradigms. Using SPSS 22, a principal components analysis (PCA) was conducted using varimax rotation on the six capture scores for Experiment 1. This analysis revealed that three underlying components could account for $60.7 \%$ of the variance in the data (see Table 4). Factor 1 appeared to represent capture by onsets, consistent with a bottom-up factor. Our measure of onset capture derived from the top-down contingent cuing paradigm and measure of bottom-up capture derived from the irrelevant singleton paradigm both loaded highly onto Factor 1. However, capture scores from the additional singleton paradigm did not load onto this factor as one might expect if this represents a measure of bottom-up capture. Factor 2 appeared to represent a top-down factor; top-down measures derived from the contingent cuing and contingent blink paradigms loaded highly onto Factor 2. Note that one loads positively and one loads negatively because in the contingent blink is largely accuracy based whereas the contingent cuing task is a speeded task. The additional singleton paradigm loaded highly onto Factor 3. While it is often assumed that onsets and color singletons capture attention in much the same, stimulus-driven way, these results suggest this may not be the case (see Boot et al., 2005, for another case in which capture by onsets and color singletons differ).

For Experiment 2, a similar PCA analysis with varimax rotation revealed that two underlying components could account for $62.3 \%$ of the variance in the data (see Table 4). Both capture measures calculated from the contingent cuing paradigm (i.e., one top-down measure, one bottom-up measure) loaded highly onto Factor 1. This is unexpected, as the onset would not be expected to capture attention in the same way as a color singleton matching the target. Factor 2 appeared to be indicative of a bottom-up factor, as both the irrelevant singleton paradigm and additional singleton paradigm capture measures loaded highly onto this factor. Thus Experiment 2 provides some support for these two bottom-up factors measure the same thing. However, the overall picture provided by the PCAs conducted on the data from Experiments 1 and 2 are not entirely consistent with the idea that capture is a unitary construct or that capture is neatly divided between bottom-up and top-down effects.

\section{Individual differences in capture}

Next, we further explored the individual differences in capture to gain additional insight into these common paradigms. Bottom-up proponents would argue that capture is purely stimulus driven and automatic. If this were the case, we would expect bottom-up capture measures to be robust, with most participants demonstrating patterns of response consistent with stimulus-driven capture. Figures 11 and 12 present histograms of all the bottomup and contingent-capture scores. As can be seen in Fig. 11, capture across bottom-up measures was not consistently observed (i.e., some participants showed anticapture, or a benefit from distracting information). 


\section{EXPERIMENT 1}
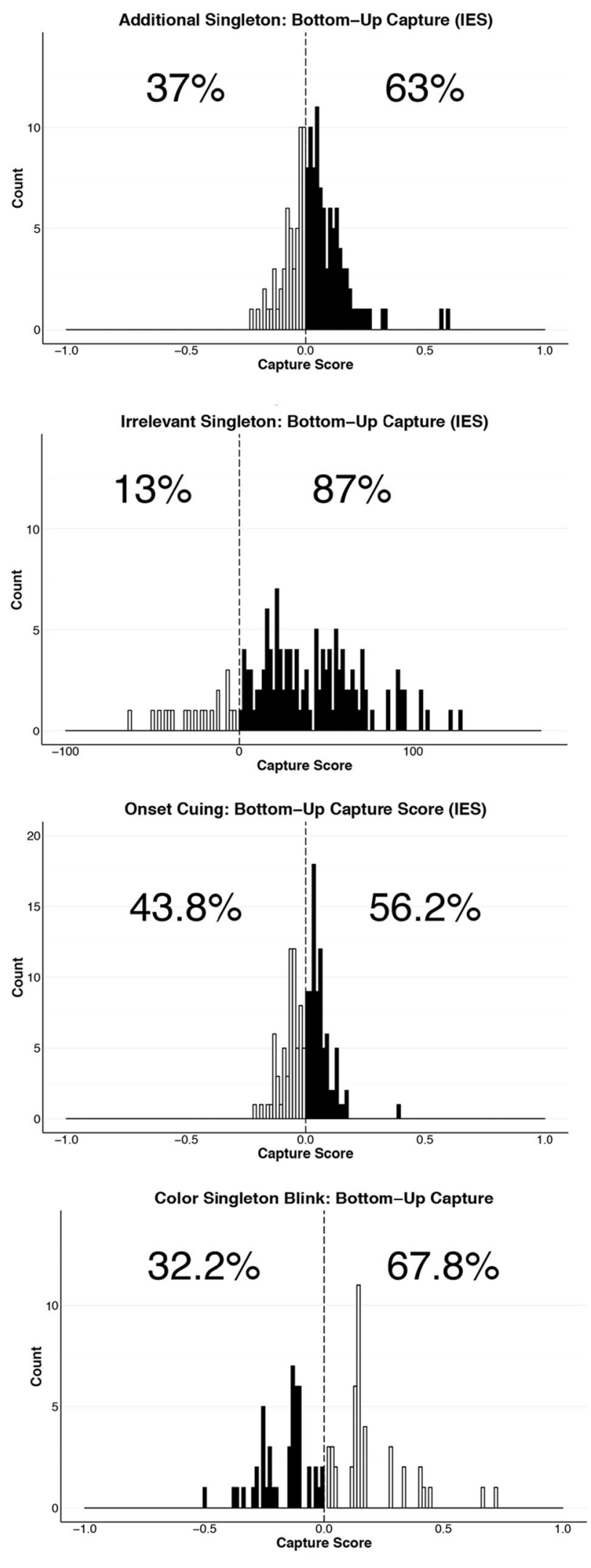

\section{EXPERIMENT 2}
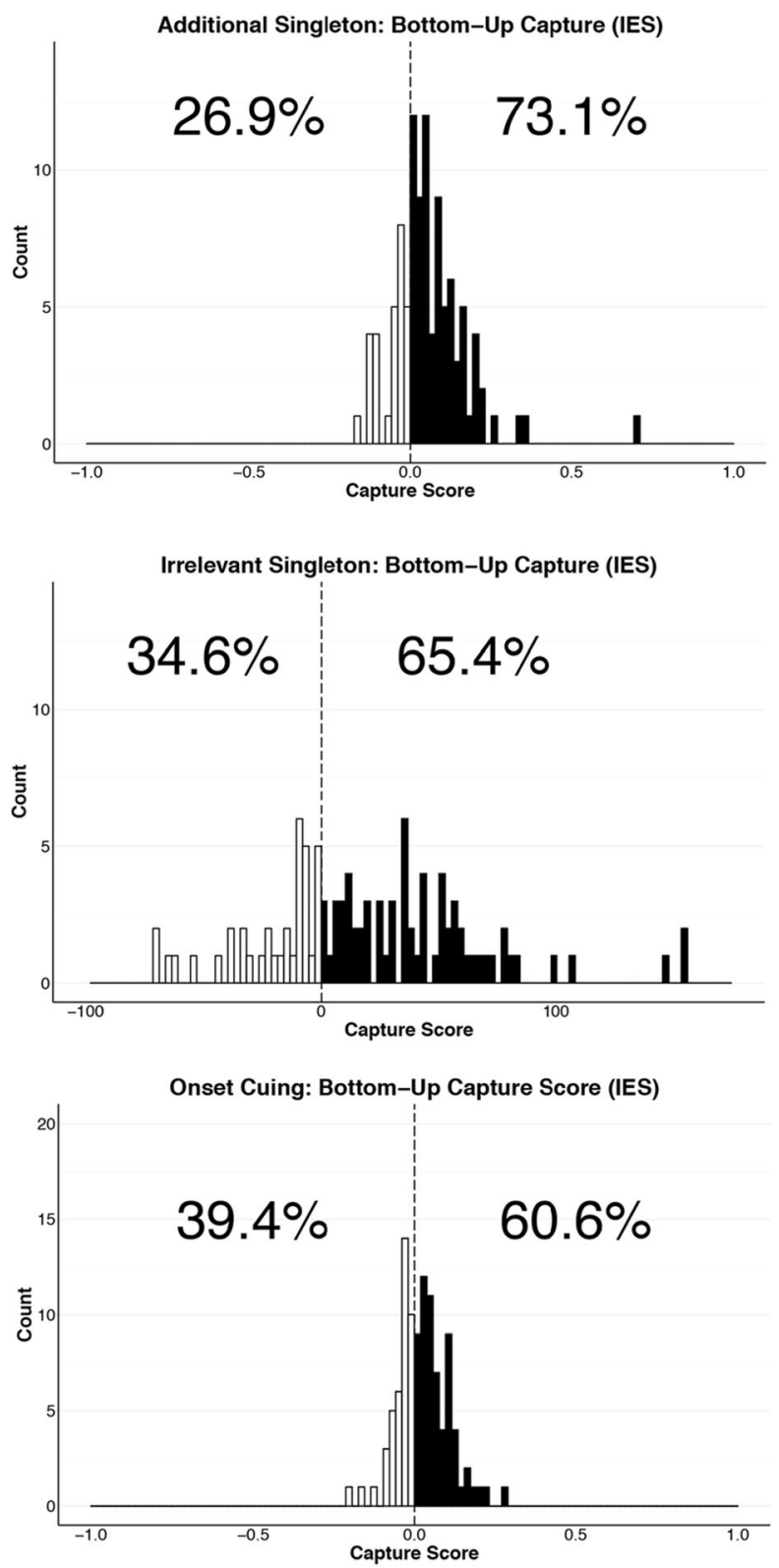

ANTI-CAPTURE 
Fig. 11 Histograms illustrating the capture differences among participants for the bottom-up capture measures. Black bars represent participants who demonstrated a pattern consistent with attention capture, and white represents a pattern not consistent with capture. One extreme outlier is excluded in the histogram for the irrelevant singleton paradigm in Experiment 2 for ease of axis comparison across experiments. IES $=$ inefficiency scores

For example, in the additional singleton paradigm, a pattern or response consistent with capture occurred for only $66 \%$ of participants, and $86 \%$ in the irrelevant singleton paradigm (Experiment 1). Based on these results, it is difficult to support the claim that stimulusdriven capture is automatic. Instead, the more robust and reliable measures were derived from the top-down paradigms (see Fig. 12), with $90 \%$ or more of the participants showing the expected capture effects. These results have implications for how we understand capture as an automatic process.

\section{Discussion}

The present study was motivated by a desire to better understand the interrelationships between attention-capture effects derived from different paradigms. Some paradigms have consistently been used to support the view that capture represents the effect of top-down attention sets (contingent capture), while others have been used in support of capture being stimulus driven and automatic. Proponents of each view have claimed that all paradigms may in fact be tapping the same capture mechanism (bottom-up paradigms really measure a form of contingent capture, or vice versa). If these tasks are in fact measuring the same construct, one would expect strong correlations. The primary, surprising finding was a lack of a relationship between tasks purporting to measure similar constructs (see also, Kawahara \& Kihara, 2011). Instead, weak or no correlations were observed in two relatively large-sample studies (see Figs. 9 and 10).

The reliability of commonly used capture scores was assessed and supported the idea that some capture paradigms may not produce stable measures of capture, and some measures of capture may be more reliable than others. The reliability of these measures is of particular importance because some of these tasks have been used to study individual differences. For example, recent studies have explored capture as a function of video-game experience (Chisholm, Hickey, Theeuwes, Kingstone, 2010) and depression (Bredemeier et al., 2012). If capture, as measured by these tasks, is unstable, then it is worth examining factors that might boost reliability in these paradigms and the number of trials necessary to produce reliable estimates of capture.

The most robust capture measures (i.e., more consistent reliability in Experiment 2, most participants demonstrating a pattern consistent with capture) were derived from topdown paradigms: the contingent blink and contingent cuing paradigms. More robust capture and greater reliability may be due to these tasks being more constraining of strategies by providing participants more information about where the target might occur and limiting display duration. For the contingent blink task, the greater reliability of its capture metric may have been due to the spatial certainty of the target (i.e., the target was always in the center of the screen). This task did not involve a spatial search process that might introduce greater variability in search strategy, with this variability influencing the degree to which attention might be captured within and between participants. Additionally, in this task, target and distractor positions were fairly stable from trial to trial compared to the bottom-up tasks in which target and distractor positions were randomized. Greater similarity between trials may result in greater reliability (with differences between trials introducing noise, resulting in less reliability). When task demands are more clearly defined and trials are more similar to one another, as in most top-down paradigms (i.e., display size and target certainty), it is possible that there is little room for strategy differences to have an influence. In bottom-up tasks where demands are unclear (i.e., lack of target location certainty), the influence of strategy differences may play a larger role.

From a theoretical perspective, the correlation between capture in the additional singleton paradigm and contingent cuing paradigm in Experiment 1 is interesting, as it has been proposed that capture in the additional singleton paradigm might reflect a top-down attention set for unique items (a uniquely colored item captures attention because participants are set to detect a unique shape). A correlation between these two tasks might reflect that attention sets underlie capture in both paradigms. However, this effect was not strong, and did not replicate in Experiment 2. Furthermore, caution should be exercised in interpreting the capture effect from the additional singleton paradigm in Experiment 1, given the unusual pattern of results (no capture at the small set size).

The greater reliability of capture in contingent paradigms, and the greater proportion of participants who demonstrated capture in these paradigms, is also theoretically interesting. While it is often assumed that bottom-up paradigms measure capture that is stimulus driven and automatic, the distributions of individual differences in capture scores observed suggested that some participants were largely unaffected by the presence of salient but irrelevant information. If, as some have proposed, bottom-up paradigms really measure the effects of subtle or general attention sets (e.g., Atchley, Kramer, \& Hillstrom, 2000; Gibson \& Kelsey, 1998), it may be that capture in contingent capture paradigms represents a stronger form of top-down capture that is also operating in "bottomup" paradigms. For example, if onsets capture attention because the display-wide onset of the search display provides 


\section{EXPERIMENT 1}
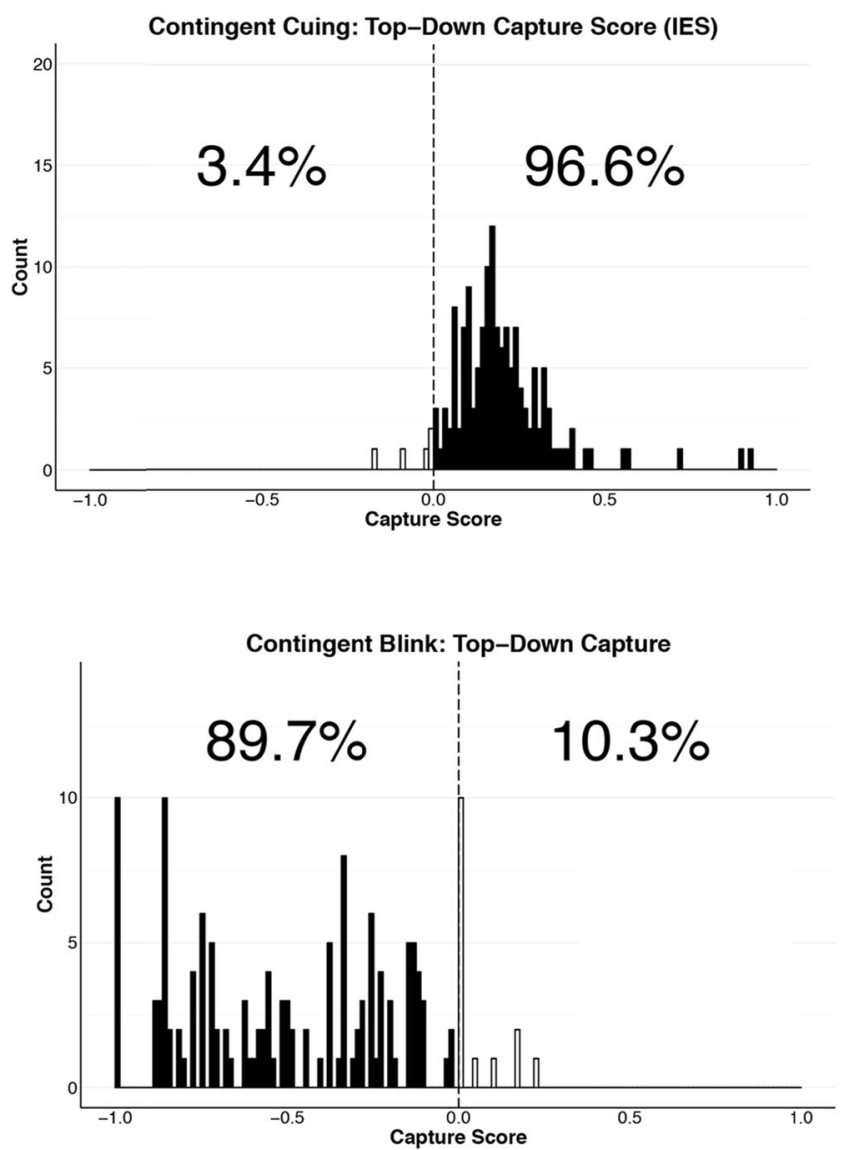

EXPERIMENT 2

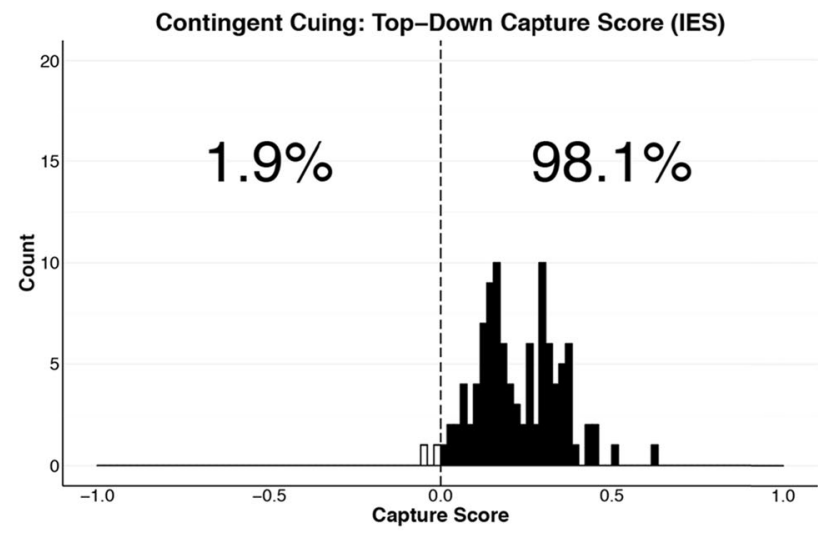

ANTI-CAPTURE

Fig. 12 Histograms illustrating the capture differences among participants for the contingent capture measures. Black bars represent participants who demonstrated a pattern consistent with attention capture, and white represents a pattern not consistent with capture

useful information as to when to begin searching, this attention set would likely not be as strong, and capture would not be as robust, compared to if an onset signaled the location of the target. The degree to which attention sets operate in search and cuing paradigms, producing robust capture effects, may depend on the temporal and spatial precision of these cues with respect to predicting when and where the target will occur.

Finally, in Experiment 1, capture in the additional singleton paradigm did not load highly onto a factor that seemed to measure stimulus-driven capture by onsets (although this pattern was not observed in Experiment 2). Additional work might explore whether, even in the same paradigm, onsets and color singletons capture attention differently. For example, Boot et al. (2005) found that a dual-task eliminated onset capture, but increased capture by color singletons in the same search task (irrelevant singleton paradigm). The same pattern was observed by Bredemeier et al. (2012) in a different paradigm (additional singleton). These dual-task results suggest that capture by color singletons and onsets may be the result of different mechanisms. Examining the correlation in capture between different types of singletons, even within the same paradigm, may provide additional insight into the nature of capture. In general, there may be benefits to using more similar paradigms (in terms of display, timing, stimuli, and task demands) to better understand the relationship between different types of capture, compared to the very different paradigms tested here and typically used to study capture.

Future work in this area may benefit from the use of verbal protocol analysis (either online or retrospective) to help tease apart possible strategy differences between 
tasks and among observers in attention-capture paradigms (Ericsson \& Crutcher, 1991). These differences might relate to task reliability and the degree of capture observed. Knowing more about how each task is approached in general, and how individuals differ in how they approach each task, may benefit our understanding of attentional control. In sum, results demonstrate that, even for relatively simple laboratory measures of attention, there are still important gaps in knowledge regarding what these paradigms measure and how they are related.

\section{References}

Atchley, P., Kramer, A. F., \& Hillstrom, A. P. (2000). Contingent capture for onsets and offsets: Attentional set for perceptual transients. Journal of Experimental Psychology: Human Perception and Performance, 26(2), 594-606.

Bacon, W. F., \& Egeth, H. (1994). Overriding stimulus-driven attentional capture. Perception \& Psychophysics, 55, 485-496.

Blakely, D. P., Wright, T., Dehili, V. M., Boot, W. R., \& Brockmole, J. R. (2012). Characterizing the time course and nature of attentional disengagement effects. Vision Research, 56, 38-48.

Boot, W. R., \& Brockmole, J. R. (2010). Irrelevant features at fixation modulate saccadic latency and direction in visual search. Visual Cognition, 18(4), 481-491.

Boot, W. R., Brockmole, J. R., \& Simons, D. J. (2005). Attention capture is modulated in dual-task situations. Psychonomic Bulletin \& Review, 12(4), 662-668.

Bredemeier, K., Berenbaum, H., Brockmole, J. R., Boot, W. R., Simons, D. J., \& Most, S. B. (2012). A load on my mind: Evidence that anhedonic depression is like multi-tasking. Acta Psychologica, 139, 137-145.

Chisholm, J. D., Hickey, C., Theeuwes, J., \& Kingstone, A. (2010). Reduced attentional capture in action video game players. Attention, Perception, \& Psychophysics, 72, 667-671.

Ericsson, K. A., \& Crutcher, R. J. (1991). Introspection and verbal reports on cognitive processes - Two approaches to the study of thinking: A response to Howe. New Ideas in Psychology, 9(1), 57-71.

Faul, F., Erdfelder, E., Buchner, A., \& Lang, A.-G. (2009). Statistical power analyses using $G^{*}$ Power 3.1: Tests for correlation and regression analyses. Behavior Research Methods, 41, 1149-1160.
Folk, C. L., Leber, A. B., \& Egeth, H. E. (2002). Made you blink! Contingent attentional capture produces a spatial blink. Perception \& Psychophysics, 64(5), 741-753.

Folk, C. L., \& Remington, R. (1998). Selectivity in distraction by irrelevant featural singletons: Evidence for two forms of attentional capture. Journal of Experimental Psychology: Human Perception \& Performance, 24, 847-858.

Folk, C. L., Remington, R. W., \& Johnston, J. C. (1992). Involuntary covert orienting is contingent on attentional control settings. Journal of Experimental Psychology: Human Perception and Performance, 18(4), 1030.

Franconeri, S. L., \& Simons, D. J. (2003). Moving and looming stimuli capture attention. Perception \& Psychophysics, 65(7), 999-1010.

Gibson, B. S., \& Kelsey, E. M. (1998). Stimulus-driven attentional capture is contingent on attentional set for displaywide visual features. Journal of Experimental Psychology: Human Perception and Performance, 24(3), 699-706.

Jonides, J., \& Yantis, S. (1988). Uniqueness of abrupt visual onset in capturing attention. Perception \& Psychophysics, 43(4), 346-354.

Kawahara, J. I., \& Kihara, K. (2011). No commonality between attentional capture and attentional blink. The Quarterly Journal of Experimental Psychology, 64(5), 991-1008.

Leber, A. B., \& Egeth, H. E. (2006a). Attention on autopilot: Past experience and attentional set. Visual Cognition, 14, 565-583.

Leber, A. B., \& Egeth, H. E. (2006b). It's under control: Top-down search strategies can override attentional capture. Psychonomic Bulletin \& Review, 13(1), 132-138.

Mathôt, S., Schreij, D., \& Theeuwes, J. (2012). OpenSesame: An opensource, graphical experiment builder for the social sciences. Behavior Research Methods, 44(2), 314-324.

Peirce, J. W. (2007). PsychoPy-Psychophysics software in Python. Journal of Neuroscience Methods, 162(1), 8-13.

Theeuwes, J. (1992). Perceptual selectivity for color and form. Perception \& Psychophysics, 51(6), 599-606.

Theeuwes, J. (1994). Stimulus-driven capture and attentional set: Selective search for color and visual abrupt onsets. Journal of Experimental Psychology: Human Perception and Performance, 20, 799-806.

Theeuwes, J. (2010). Top-down and bottom-up control of visual selection. Acta Psychologica, 123, 77-99.

Theeuwes, J., Kramer, A. F., Hahn, S., \& Irwin, D. E. (1998). Our eyes do not always go where we want them to go: Capture of the eyes by new objects. Psychological Science, 9, 379-385.

Townsend, J. T., \& Ashby, F. G. (1983). Stochastic modeling of elementary psychological processes. Cambridge: Cambridge University Press.

Yantis, S., \& Jonides, J. (1984). Abrupt visual onsets and selective attention: Evidence from visual search. Journal of Experimental Psychology: Human Perception \& Performance, 10, 601-621. 